\title{
Seismic Sequence Structure and Earthquakes Triggering Patterns
}

\author{
Giulio Riga1, Paolo Balocchi ${ }^{2}$ \\ ${ }^{1}$ Geologist, Independent Researcher, Lamezia Terme, Italy \\ ${ }^{2}$ Geologist, Independent Researcher, Modena, Italy \\ Email: giulio.riga@tin.it
}

Received 31 December 2015; accepted 15 February 2016; published 18 February 2016

Copyright (C) 2016 by authors and Scientific Research Publishing Inc.

This work is licensed under the Creative Commons Attribution International License (CC BY). http://creativecommons.org/licenses/by/4.0/

(c) (i) Open Access

\section{Abstract}

Within a time distribution of magnitude values, before any mainshock some earthquake triggering patterns with several features develop, under tectonic processes' influence, through which it is possible to early identify the preparation phase of big earthquakes. The purpose of this article was to identify and classify the warning patterns that develop before a big earthquake by considering space-time seismicity variations. The methodological approach adopted was of graphical type, based on procedures of technical analysis currently used to estimate the financial markets. In the initial phase of the study we have analyzed the seismic sequences types described in the bibliography (type 1: foreshocks-mainshock-aftershocks, type 2: mainshock-aftershock; type 3: swarm) and the main structure of the seismic cycle, within which maximum and minimum magnitude values characterize the pattern that it develops until the main event changes. Then, we assessed the position of foreshocks, mainshock and aftershocks within the seismic cycle in order to identify the warning pattern that characterized the exact time when the energy emission occurs. As to the evolution normally shown over time, we have grouped the warning patterns in 2 categories: 1) progressive earthquake pattern; 2) flash earthquake pattern. Finally, we have made a classification of the warning pattern related to the fluctuations of maximum and minimum magnitude values, compared its form with the mainshock's focal mechanism and suggested some graphic procedures in order to estimate the mainshock magnitude value associated with each warning pattern. The results we obtained unquestionably allow a better comprehension of preparation process of a large earthquake, improving the earthquakes forecasting probability in the next future.

\section{Keywords}

Foreshock, Classification, Sequence, Cycle, Pattern, Trigger, Earthquake 


\section{Introduction}

The analysis of the seismic sequence structures underlines (points out) that magnitude values do not unpredictably change, but follow some trends that can be identified in a chart.

Starting from this principle, we can speculate that some behaviors cyclically recur, making it possible, if identified on time, for the formulation of hypothesis about the future evolution of the seismic sequence.

The checks we have performed on several big earthquakes occurred in the last 40 years, have highlighted that it is possible to identify some earthquakes triggering patterns in the short and medium term, whose space-time complexity is strictly linked to the following items: a) the seismic region heterogeneity [1]; b) the stress state inside and around the source of a big earthquake [2] [3]; c) the long-range interactions among different seismic and heterogenic regions.

The identification, in advance, of the warning pattern within the seismic sequence, provides several useful insights to understand what is happening in the source region area, which can be used to improve earthquakes predictions.

Below, we will explain the known seismic sequences features, which include warning patterns we have classified and discussed in relation to the magnitude values' trend.

We performed the analysis of the seismic sequence using: a) spatial coverage of datasets ranging from portions of a region to several countries (from a few tens of kilometers to thousands of kilometers); b) magnitude range of $2.0-10 \mathrm{M}$; c) depths range of $1-50 \mathrm{~km}$.

\section{Method of Analysis}

We analyzed and classified the time series of magnitude values using the results of some researches carried out in the field of financial markets studies.

The analysis provides some characteristics of the values' trend in a time series which are useful for assessing whether the phenomenon occurs according to the predicted scheme.

In particular, for the study of the movements of the seismic cycle we used the Elliott's wave theory [4]-[6], where the concept of pattern is one of the most significant aspects.

The methodological approach adopted for the classification of earthquake triggering patterns and the assessment of the main earthquake magnitude is based on the technical analysis [7] [8], which consists in a methodology for the evaluation of financial markets used to identify trends and assess future trends possibility. Similarly, it may be used as a safe tool to analyze the most significant magnitude values' oscillations and to predict likely future events, mainly through graphical methods and statistics.

\section{Structure of Seismic Sequence}

\subsection{Types of Seismic Sequences}

Earthquakes are generally part of a sequence with variable and sharp features. Mogi [9] divided these sequences in three types: 1) mainshock-aftershock (Figure 1(a)); 2) foreshock-mainshock-aftershock (Figure 1(b)); 3) swarm (Figure 1(c)). The foreshocks and aftershocks sequences are strictly associated to a bigger event called mainshock, while the swarm is a sequence of earthquakes and is not associated with a dominant earthquake. The subsequent events of mainshock-aftershock sequences of similar dimension (Figure 1(d)) are defined as swarm, but they clearly differ from an ordinary swarm (Figure 1(c)).

Moreover, numerical results [9] show that different homogeneity index will produce different patterns of seismic sequences. We have three types of sequences: a) swarm; b) foreshock-mainshock-aftershock; c) mainshock-aftershock that happen specifically in these cases: a) extremely heterogeneous; b) mildly heterogeneous and c) homogeneous [1].

Isolated earthquakes occur in presence of a great pre-stress and/or low stress at break. Foreshocks-mainshock-aftershock seismic sequences happen in presence of a mild pre-stress and stress at break. Swarms happen in case of low pre-stress and/or high resistance to break. Very low values of pre-stress and/or very high break strength are the best conditions for the aseismic creep event [10] to occur. Other studies underline that in areas where the time variation of the strain-rate (measured by geological surveys GPS) is low, higher magnitude earthquakes are expected compared with areas with high strain-rate [11].

Simulations [10] suggest that an isolated earthquake can be interpreted as the extension of a break in a 


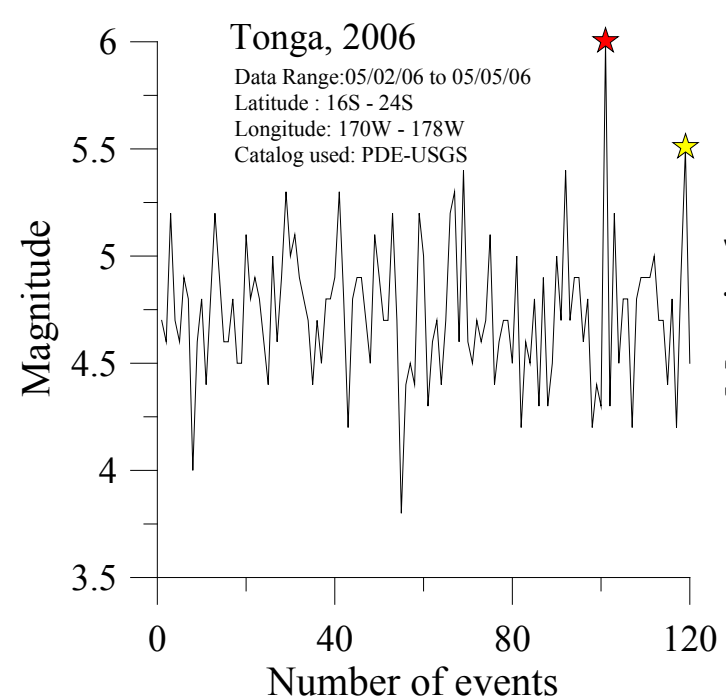

(a)

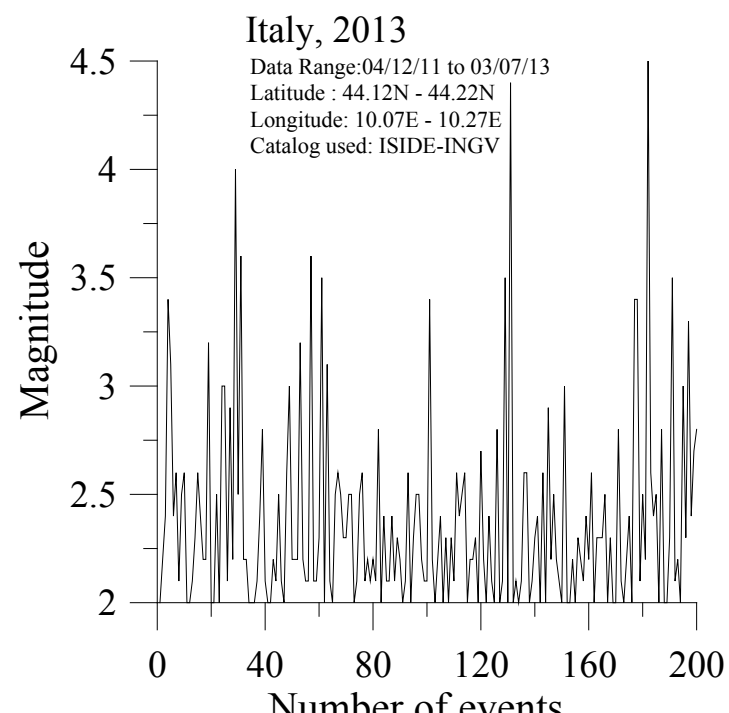

Number of events

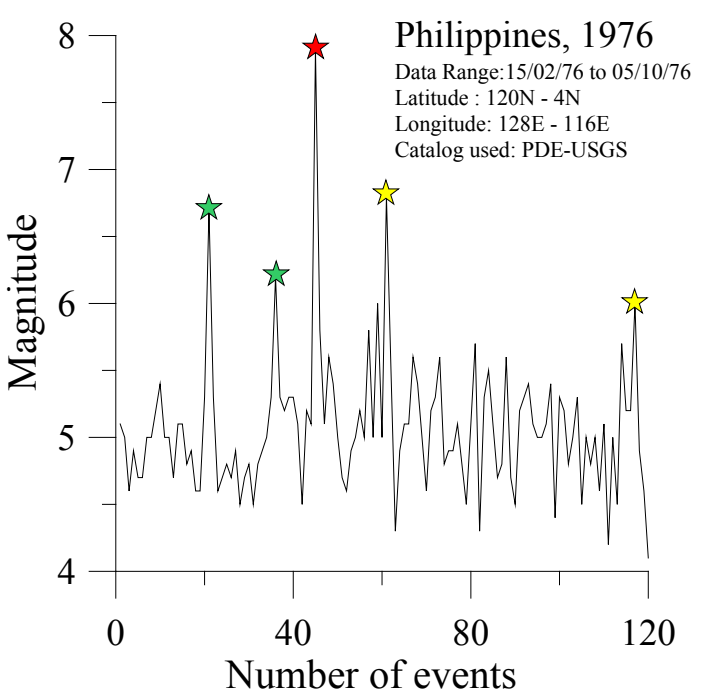

(b)

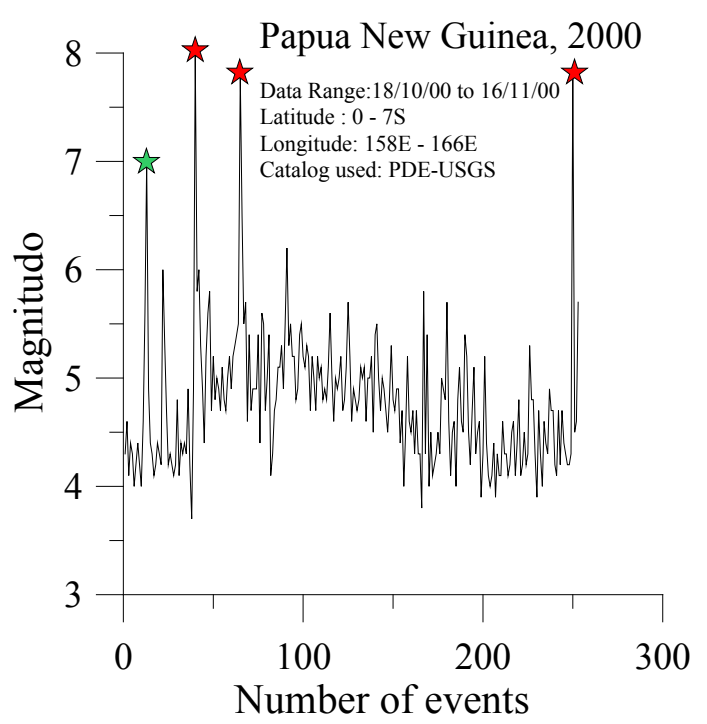

(d)

Figure 1. Examples of earthquake sequences: (a) mainshock-aftershock sequence; (b) foreshock-mainshock-aftershock sequence; (c) earthquake swarm; (d) subsequent occurrence of mainshock-aftershock sequence.

non-uniform field, while a foreshocks-mainshock-aftershocks sequence results from a break extension to a nonuniform field having modest fluctuations and swarms are a consequence of a break extension to a very inhomogeneous sector.

Swarms are a sequence of earthquakes that often start and finish gradually, where there is no evidence of any earthquake shock with higher magnitude [12]. However, shocks that make up swarms are actually included in seismic cycles in which the main shock, usually of mild magnitude, occurs always at the end of the nucleation process.

Swarms are divided in two typologies. The first one is the ordinary swarm in which the activity changes fairly irregularly (Figure 1(c)), and has a duration between some hours and little more than a year. Most of the swarms happening in volcanic zones show this model, which reflects the magmatic activity in depth. The second typology features a succession of main shocks with similar magnitude (Figure 1(d)) whose duration is between several days and months. 


\subsection{Seismic Cycle's Basic Structure}

The long-term seismic sequence consists of several cycles that are repeated according to the representation scale and whose duration varies from a few minutes to several years.

The basic seismic cycle is a collection of processes that develop before, during and after an earthquake, which are highlighted by the variations in the magnitude values that develop in short and medium term time windows. The term "cycle" suggests that big earthquakes can be either periodic or regular events over time. The analysis of the succession of the seismic cycles included in a seismic sequence shows that the periodicity can be prevalent among big earthquakes recorded in areas even with different extension.

According to the magnitude values fluctuations, seismic cycles can be divided in four stages: 1) trigger; 2) energy release; 3) breaking; 4) adjustment (Figure 2).

Trigger stage. In this phase, the magnitude values vary within a very close range in which a small shock, after some fluctuations, triggers the next one.

Energy release stage. This step follows the trigger phase, where the magnitude values fluctuations produce increasing maximum values along with an increase in the number of shocks. In type 1 sequences, (mainshockaftershock) these characteristics are often not found.

Breaking stage. This is the energy release phase's top. It corresponds to the main breaking point i.e. when the mainshock occurs, generally marked by the highest number of recorded shocks. If no additional main breaking (multiple events) follows, the magnitude values fall quickly under the peak magnitude value recorded.

Adjustment stage. The magnitude values and the number of shocks decrease, until it gets back to the trigger phase once again.

The transition from the energy release phase to the adjustment one can be confirmed by the values decrease before and after the mainshock, called "transition line" (Figure 3(a)).

We should bear in mind, however, that usually after being dropped below the "transition line" (point 3), the magnitude values temporarily return to the same line level or even slightly above, before definitely starting to decrease up to the next minimum value.

In case of "transition line" crossing during the increase phase, a second mainshock may occur (subsequent occurrence of mainshock-aftershock sequences).

In the case of minimum " 1 " and " 2 " descending values, the step from energy release to the adjustment one is always confirmed by the minimum "2" value formation (Figure 3(b)).

\subsection{The Seismic Cycle Movements}

In many long-term seismic sequences, the analysis of magnitude values trends during the energy release phase shows a cyclical fluctuation, which is characterized by one or more waves (foreshocks-mainshock). In the adjustment phase, we observe several aftershocks that partially rebalance all the impulsive movement in the previous step.

Based on Elliot's theory [4]-[6], a seismic sequence can be considered as a succession of cyclic variations of magnitude values whose duration may vary over time (Figure 4 and Table 1), where the most energetic seismic

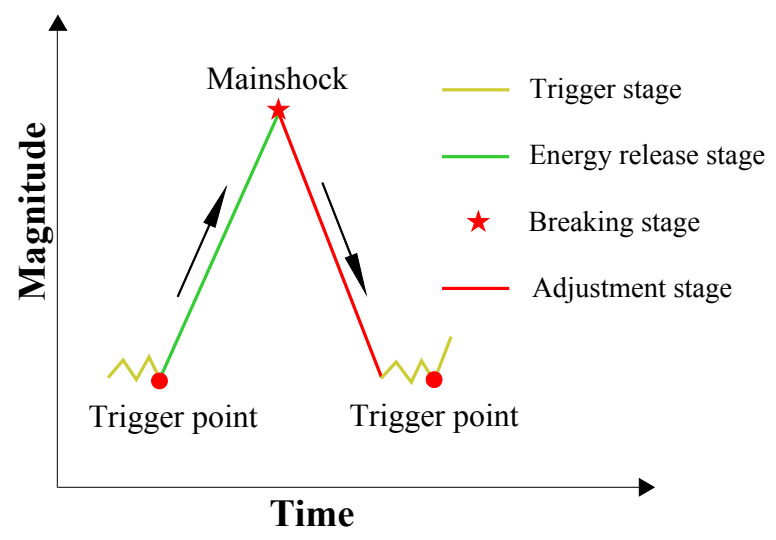

Figure 2. Schematic representation of the seismic cycle's four stages. 


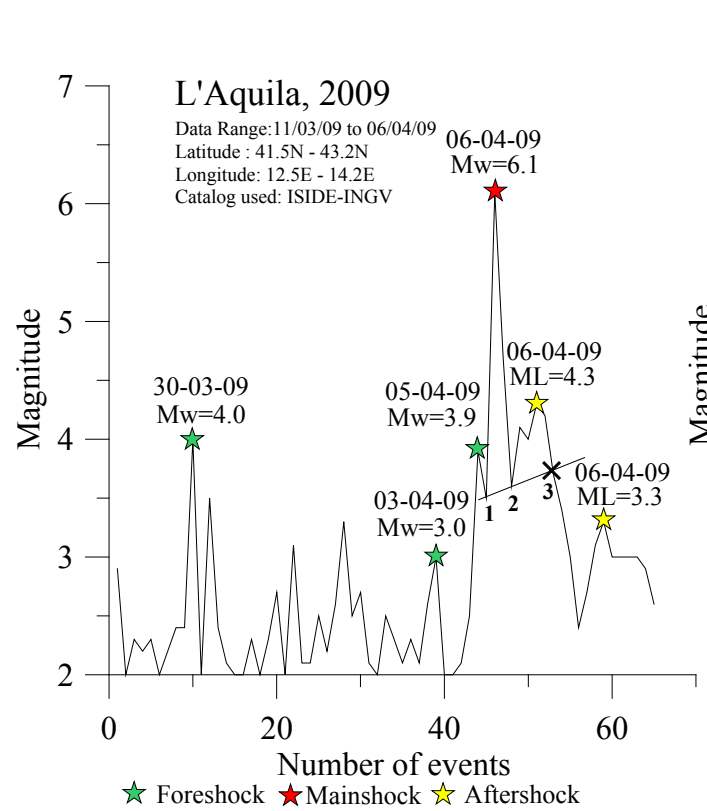

(a)

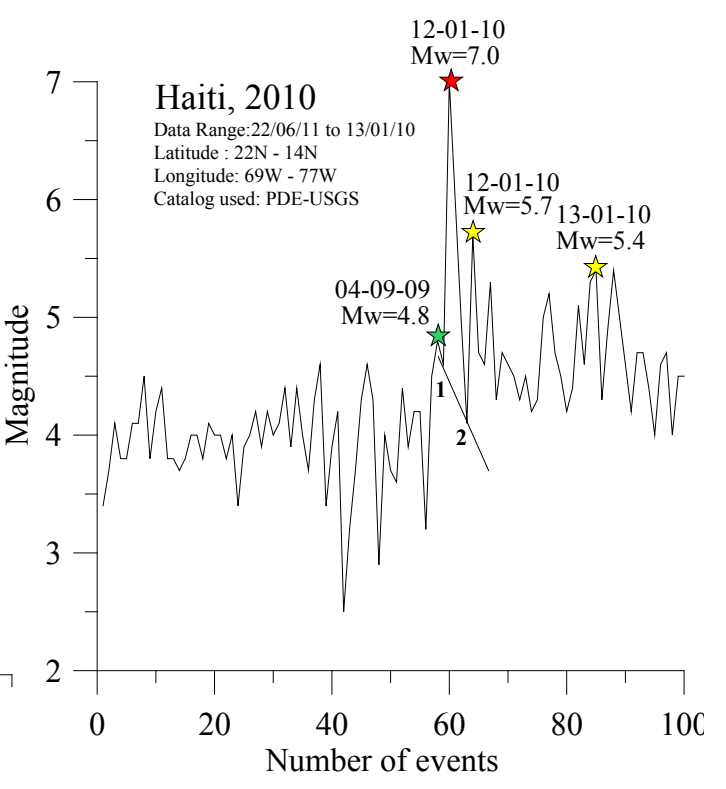

(b)

Figures 3. Sequence of earthquake imminent period: (a) L'Aquila earthquake, 06-04-2009 (Italy); (b) Haiti earthquake, 12-01-2010.

- Primary trigger point

$\Delta$ Secondary trigger point

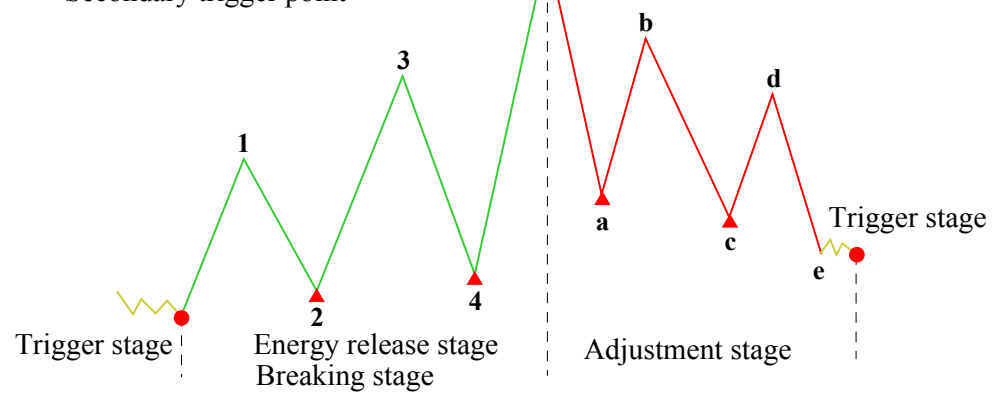

Figure 4. Schematic representation the seismic cycle's waves.

Table 1. Seismic cycle-energy release and adjustment phases' waves.

\begin{tabular}{ccc}
\hline Point & Waves & Description \\
\hline 1 & Energy release & Formation of a first foreshock \\
2 & Adjustment & Formation of a relative minimum \\
3 & Energy release & Formation of a second foreshock \\
4 & Adjustment & Formation of a relative minimum \\
5 & Energy release & Formation of a mainshock \\
$\mathrm{a}$ & Adjustment & Formation of a relative minimum \\
$\mathrm{b}$ & Energy release & Formation of a first aftershock \\
$\mathrm{c}$ & Adjustment & Formation of a relative minimum \\
$\mathrm{d}$ & Energy release & Formation of a second aftershock \\
$\mathrm{e}$ & Adjustment & Formation of a relative minimum
\end{tabular}


events in the energy release phase occur according to a pattern consisting of several waves. Most frequently the pattern consists of five waves $(1,2,3,4,5)$ whose values in 1-3-5 show an increasing magnitude trend (foreshocks-mainshock), while in the adjustment stage it consists of five waves (a, b, c, d, e) whose magnitude values in "b" and "d" are increasing compared to the values in "a" and "c" (significant aftershocks).

The energy release stage starts after the formation of the primary trigger point, while the transition from a decreasing movement to an increasing one and vice versa is marked by a secondary trigger point.

Table 1 describes the fluctuations of the magnitude values over time and reports the description of the maximum values that are formed at the end of each wave is given as well.

The basic chart is characterized by a series of several seismic cycles, showing a nonlinear structure with an up and down progression (Figure 5) in which the different phases of the seismic process before a big earthquake are clearly visible.

Typically, a big earthquake may be preceded by two types of energy release phase: 1) progressive earthquakes and 2) flash earthquakes.

In the progressive earthquakes-type energy release phase, a big earthquake is preceded by one or more foreshocks of various kinds, while in the flash earthquakes type, a big earthquake is not preceded by any foreshock at all.

The adjustment phase consists of two or more primary aftershocks with a lower magnitude compared to the mainshock. They usually occur days, months, sometimes even years after the main event to rebalance the elastic deformation caused by the mainshock.

Figure 6(a) shows the chart of the magnitude values recorded in Japan from 1973 to 2015. Here, we can observe two similar medium-term seismic cycles. The first consists of three energy release phases and one adjustment phase. The first ended on May 8th, 1974 with a $6.7 \mathrm{Mw}$ foreshock, the second on June 12th, 1978 with a 7.7 Mw foreshock and the third on May 26th, 1983 with the $7.8 \mathrm{Mw}$ mainshock, followed by the adjustment phase which ended on November 11th, 1989 with the aftershock.

The second cycle, not completed yet, consists of the energy release phases, represented by the foreshocks occurred on 28 December 1984 (7.8 Mw) and the 25 September 2003 (8.3 Mw), while the last one, with the high energy earthquake occurred on 11 March 2011 which has not been followed by a significant aftershock yet.

Figure 6(b) shows the chart of the magnitude values recorded in Greece in 2008: here, we can observe a traditional cycle consisting of one energy release phase with two short-term foreshocks, the mainshock and one adjustment phase with two main aftershocks.

\subsection{Progressive Earthquakes Type}

Typically, the energy release phase that precedes a large earthquake consists of a series of warning shocks of

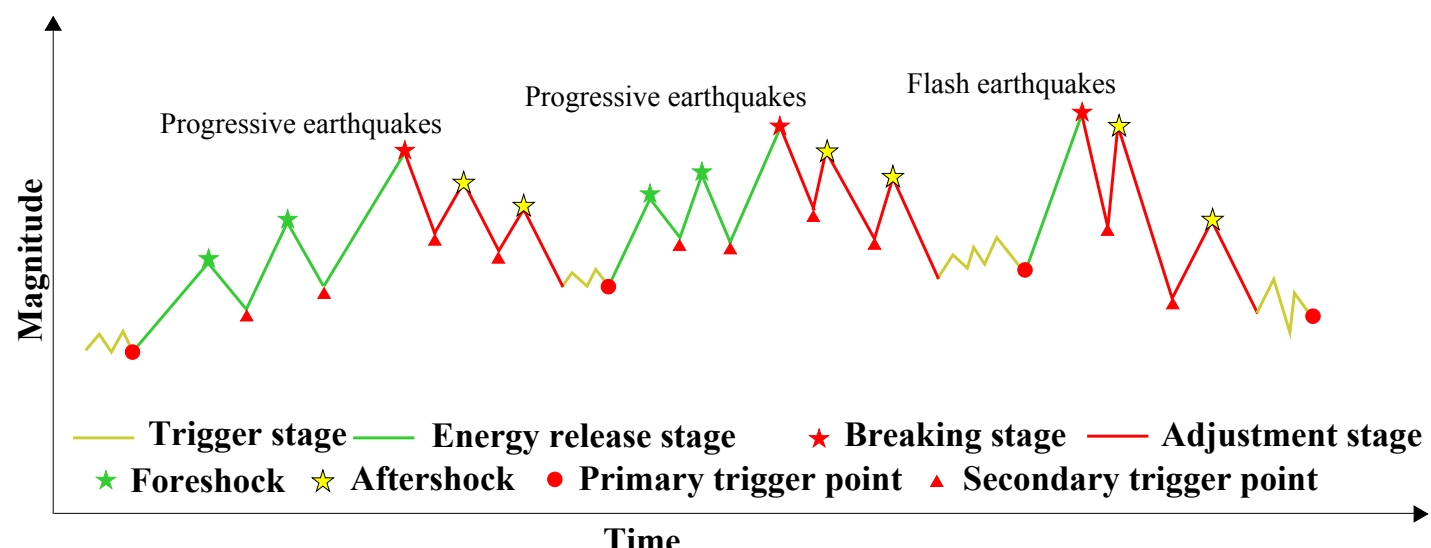

Time

T1

$\mathbf{T 2}$

$\mathbf{T} 1 \neq \mathbf{T} 2$

Figure 5. Schematic representation of the seismics cycle. The green, red and yellow-colored stars indicate the foreshocks, the mainshocks and the aftershocks, respectively. The red-colored circle indicates the energy release phase's trigger point. 


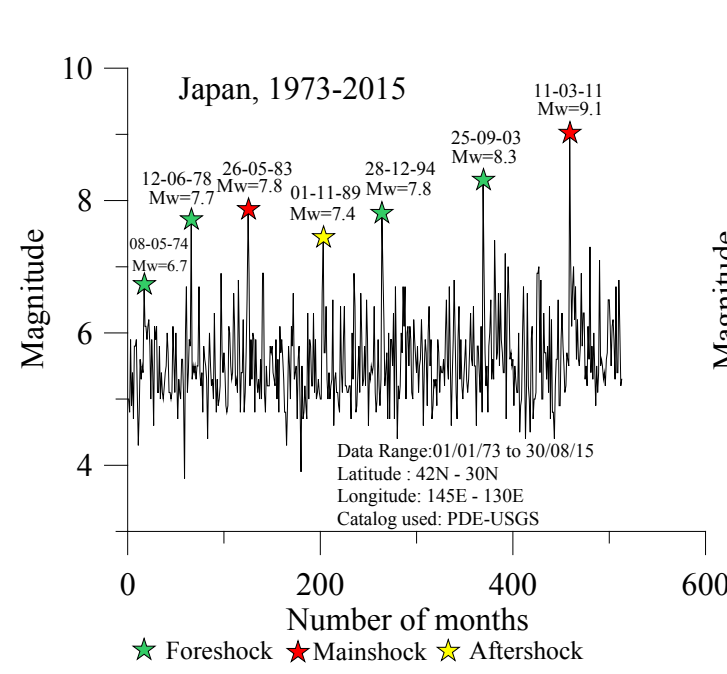

(a)

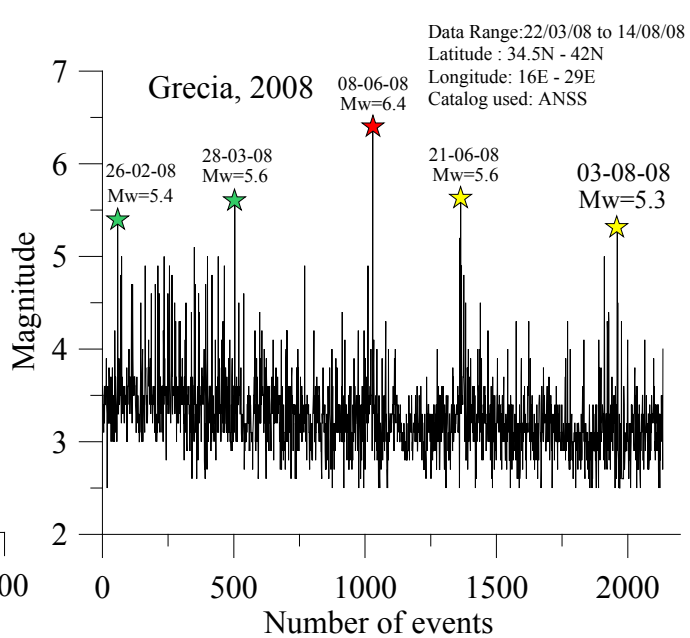

(b)

Figure 6. Examples of seismic cycles: (a) long-term sequence; (b) short-term sequence.

different order (foreshocks) featuring an increasing magnitude characterizing the breaking process that ends with a big earthquake.

It begins to develop at the end of an adjustment phase with an opposite motion compared to the preceding one.

Based on the magnitude values' fluctuations that characterize the energy release phase, it becomes clear that the greater the amplitude between two foreshocks, the greater the magnitude of the mainshock expected.

The form varies according to the temporal position and the foreshocks' magnitude values that precede the mainshock.

Depending on the foreshocks position, the pattern may consist of:

a) One or more first-order or medium-short-term foreshocks and manshock (Figure 7(a));

b) One second-order or impending term foreshock and mainshock (Figure 7(b));

c) One or more first-order or medium-short-term foreshocks, one second order or impending-term foreshock and mainshock (Figure 7(c));

d) One or more various order foreshocks and several mainshocks (Figure 7(d)).

The adjustment phase consists of two or more main aftershocks with a lower magnitude compared to the mainshock. Usually the aftershocks occur days, months, sometimes even years after the main event to rebalance the elastic deformation the mainshock caused.

Figure 8(a) shows the pattern that anticipated the earthquake occurred in Nicaragua on September 2nd, 1992. This pattern consists of two first-order foreshocks and one second-order foreshock (M 5.8) that occurred shortly before the mainshock on 2 September 1992 in the proximity of its hypocenter.

The second-order foreshock recorded on 10 August 1992 with $5.8 \mathrm{M}$, most likely indicates the beginning of the breaking phase that ended with the mainshock.

Figure 8(b) shows the pattern of the earthquake occurred in Nicaragua on September 2nd, 1992, which was processed by using a seismic area extended to the state of Guatemala. We can note how the pattern retains the same foreshocks, mainshocks and aftershocks distribution of Figure 13(a), but includes seismic events that occurred in different times and different areas.

Figure 9(a) shows the pattern that preceded the earthquake that took place in Vanuatu in 2009. Usually, only one mainshock occurs, but here the strongest earthquake was preceded on the same day (October 7th, 2009) by a high magnitude event classifiable as mainshock.

The second-order foreshock on 10 August 2009, with $6.6 \mathrm{Mw}$ magnitude, marks the beginning of the breaking phase which ended with the three strong mainshocks.

Figure 9(b) shows the pattern which preceded the earthquake in Loma Prieta (California, USA). We can see the two first-order foreshocks that preceded the mainshock on 18 October 1989 and the two main aftershocks that followed it. 


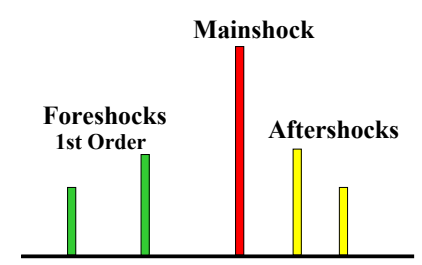

(a)

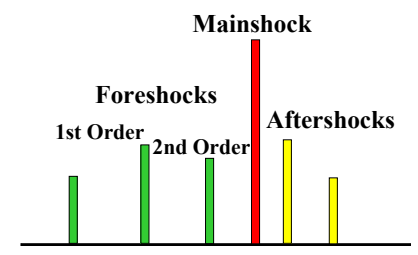

(c)

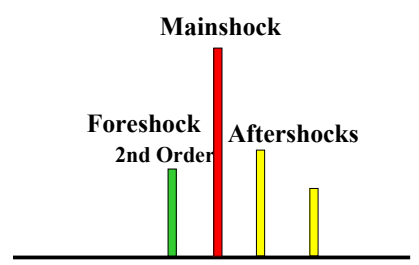

(b)

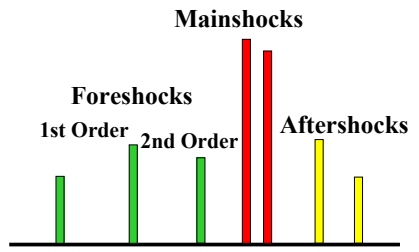

(d)

Figure 7. Schematic representation of progressive earthquakes patterns.

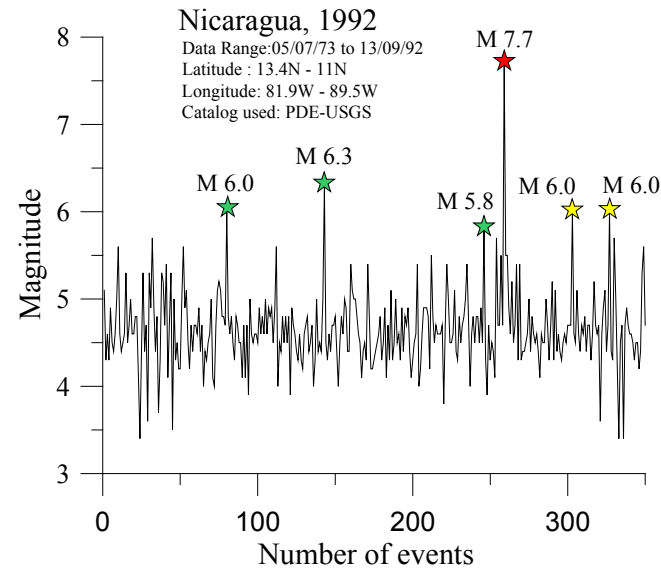

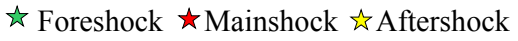

(a)

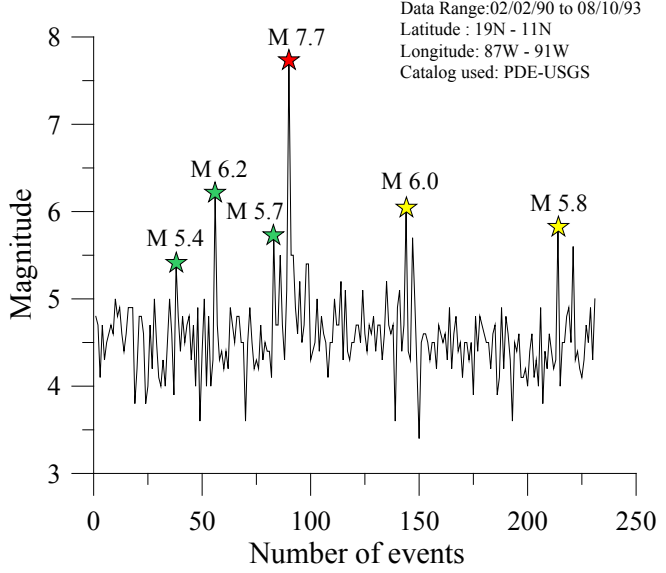

(b)

Figure 8. Progressive earthquakes patterns of Nicaragua earthquake, 1992: (a) restricted area; (b) wide area.

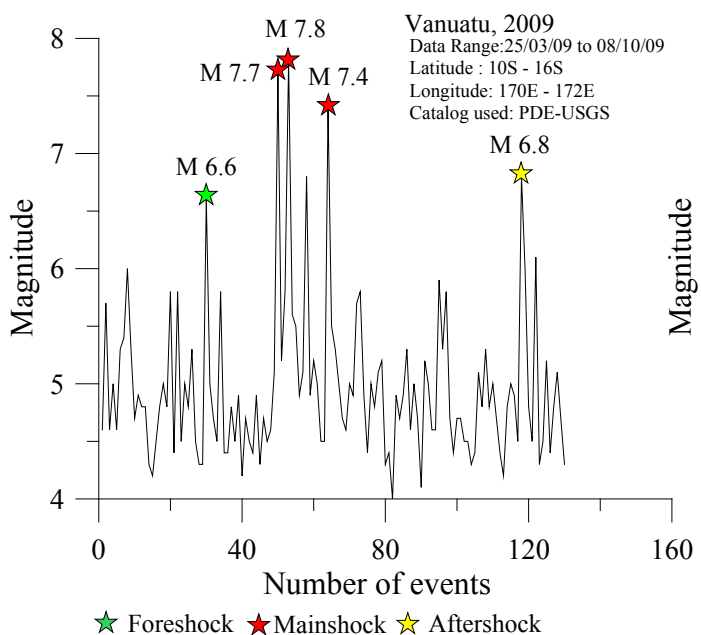

(a)

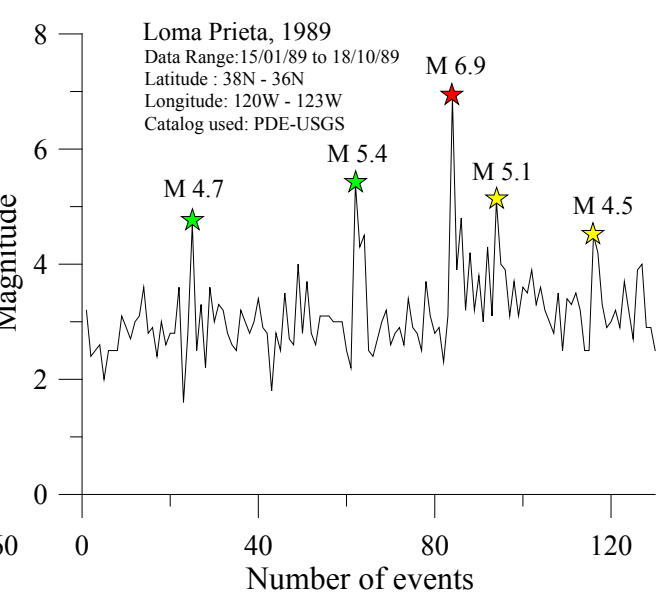

(b)

Figure 9. Progressive earthquakes patterns: (a) Vanuatu earthquake, 2009; (b) Loma Prieta earthquake (USA). 


\subsection{Flash-Type Earthquake}

This type of energy release phase is quite uncommon. Here, the big earthquake develops suddenly (with no foreshocks) after a long adjustment phase following the breaking stage and consists of aftershocks whose magnitude slowly decreases over time.

The magnitude of the expected mainshock depends on the adjustment stage time amplitude.

Based on the adjustment stage duration, it is clear that the more extended over time, the stronger the mainshock.

Depending on the aftershocks magnitude the pattern may consist of:

a) One or more significant aftershocks with decreasing magnitude (Figure 10(a));

b) One or more significant aftershocks with a similar magnitude (Figure 10(b)).

Figure 11(a) shows the pattern that preceded the earthquake in Taiwan on 20 September 1999. The maishock was followed by a first main aftershock whose magnitude was slightly lower than the mainshock's, while Figure 11(b) shows the pattern that preceded the earthquake in Tonga on 3 May 2006. Here we observe how a high magnitude mainshock was preceded by a sequence, whose magnitude values are consistent with the rest of the sequence in the post-mainshock period.

\section{Earthquake-Triggering Patterns}

The sequence of aftershocks that develops after a mainshock or a foreshock event may have a significant impact on the next earthquake triggering pattern that anticipates another mainshock formation.

The effect is more apparent in those with the longest duration that consist of aftershocks associated with a large earthquake. Some earthquakes triggering patterns demonstrate how a sequence of earthquakes can trigger other earthquakes or how small earthquakes significantly contribute to the triggering of a big earthquake.

Statistical studies performed on seismic sequences worldwide showed that pre-earthquake seismic patterns, in relation to the evolution usually occurring over time, can be broadly divided in two types:

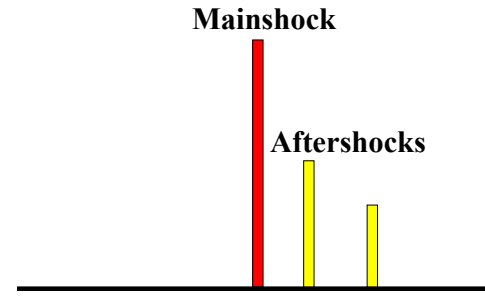

(a)

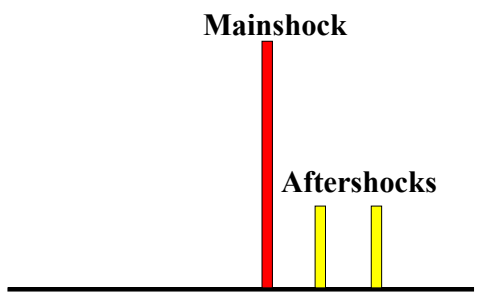

(b)

Figure 10. Flash type earthquakes' pattern illustration.

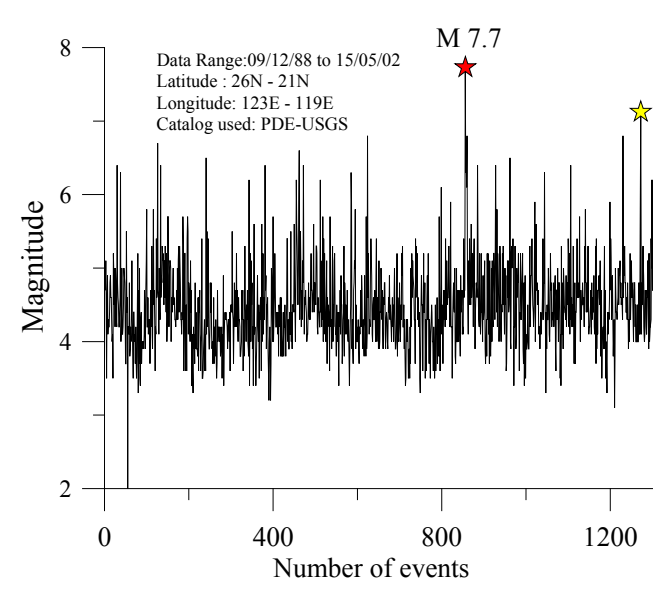

(a)

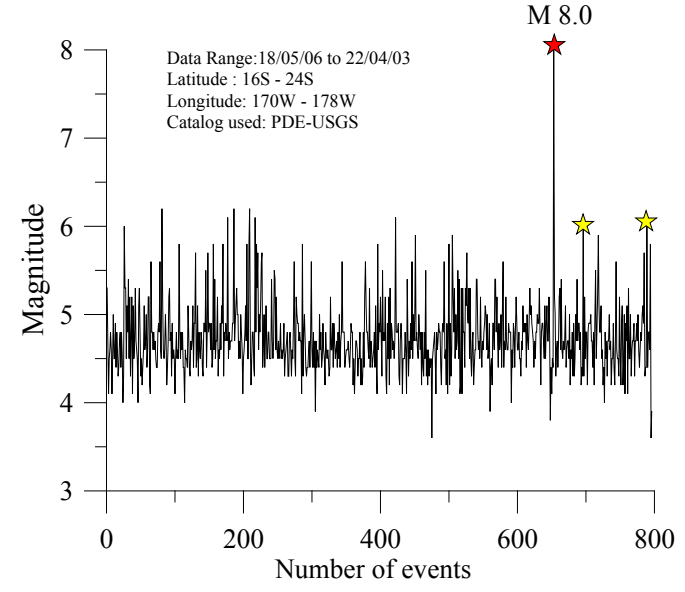

(b)

Figure 11. Examples of flash earthquales types: (a) Taiwan earthquake, 1999; (b) Tonga earthquake, 2006. 
-Flash earthquakes patterns;

-Progressive earthquakes patterns.

In flash earthquakes patterns, the magnitude values vary within two trend lines - inclined or horizontal - until the pattern reaches a critical state (breaking conditions) imposed by the rocks' strength properties and by the fault surface, where the small shock triggers the energy release phase that occurs all of sudden.

The upper trend line crossing determines the beginning of the breaking stage (breaking stage).

A small scale decrease in the crustal heterogeneity along with an increase in its depth, suggests that an increase in the normal stress inhibits the foreshocks occurrence [13].

Usually, the mainshock is followed by aftershocks that are smaller compared to the mainshock, although occasionally an aftershock can be strong enough to assume the appearance of a subsequent mainshock.

Overall, the adjustment stage appears to be extended due to the effect of a large number of events that occur after the mainshock, but first and foremost it is characterized by events with decreasing magnitude.

In particular, the number of shocks recorded from the trigger point (first minimum value) up to the mainshock is $50 \%$ smaller compared to the total number of shocks recorded up to the second minimum value formed after the first significant aftershock (Figure 12(a)).

As to the chart, the final minimum value will be placed on a lower level compared to the trigger point: this makes the pattern take an apparently sinusoidal shape with maximum decreasing values (mainshock and aftershocks).

The time this pattern needs to complete varies from a few weeks up to a number of years.

In progressive earthquakes patterns, the magnitude values oscillate within two trend lines which diverge until the main shock.

The mainshock, therefore, is preceded by foreshocks that may occur at different times both inside and outside of the source region.

The number of shocks usually used from the trigger point up to the mainshock is greater than $50 \%$ of the total number of registered shocks to reach the second minimum value that is formed after the mainshock (Figure 12(b)).

This means that, until the mainshock, the energy release phase usually is more extended than the adjustment one due to a higher number of events with increasing magnitude.

As regards the chart, the second minimum value will be higher than the Trigger point's: this makes the cycle take the apparently classical sinusoidal shape with increasing maximum and minimum values that tend to return to the initial level.

\section{Earthquake Triggering Patterns Classification}

The proposed classification is the result of a thorough graphical analysis of seismic sequences, which is the basis for the first part of the short-term, deterministic forecasting model currently in the testing phase.

The characteristics taken into consideration in the classification process are: a) the fluctuations of the magnitude minimum and maximum values; $b$ ) the foreshocks observation before the mainshock.

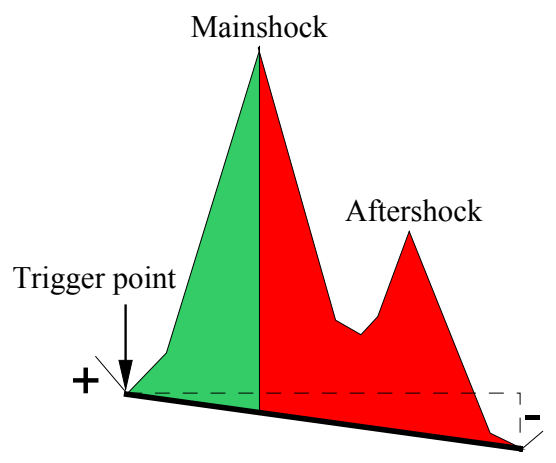

(a)

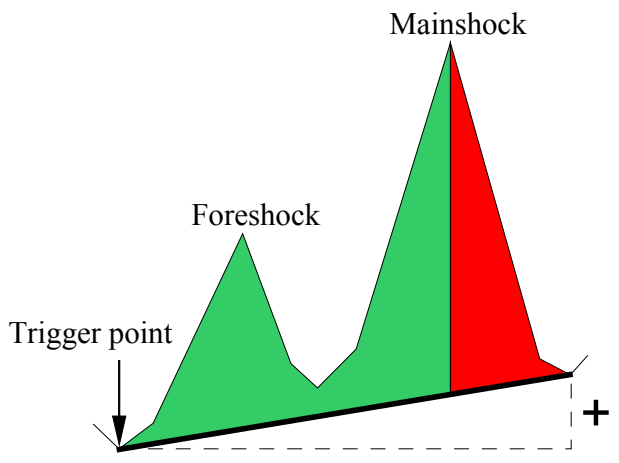

(b)

Figures 12. Schematic representation of pre-earthquake seismic types (a) flash earthquakes patterns; (b) progressive earthquakes parterns. 
The patterns identified and classified using the technical graphic analysis [7] [8], reflect the energy release and breaking phases and attempt to provide useful information to create connections between the pattern and the preparation process for a big earthquake.

\section{(A) Symmetrical triangle pattern}

The preparation process for a big earthquake starts with a first medium-strong shock, a short-medium term foreshock, followed by minor aftershocks, which are increasingly weaker and less frequent, included in a phase in which the deformation of the rock is probably minimal.

Observing this diagram in details (Figure 13), we note how the magnitude values oscillate between two trend lines that define maximum decreasing and minimum increasing values, which converge towards a triangle intersection point (Apex). The fluctuations' amplitude, which is formed inside the triangle, gradually decreases and the earthquake occurs when the magnitude values are nearing the apex. Therefore, the more extended over time the magnitude and amplitude values fluctuations, the stronger the earthquake associated with the pattern.

Usually, the critical phase begins when the difference in magnitude between points 4 and 5 is $50 \%$ less compared to the one between points 2 and 3 (triangle base). The crossing of the upper trend line currently occurs between $50 \%$ and $75 \%$ of its length.

The energy release phase may consist of the mainshock preceded by an impending term foreshock (progressive earthquakes), or by the mainshock preceded by no foreshock (flash earthquakes).

The pattern is considered as an "energy adjustment or accumulation", because the magnitude maximum values and the number of shocks decrease during the development, while increase upon the pattern breaking.

The symmetrical triangle pattern is typical of areas with little fractured, homogeneous rocks and it is most likely associated with planes of fault featuring little ruggedness.

The magnitude target (TM) of this pattern, once completed, is obtained by projecting from the breaking point (point 5), the width of the triangle base.

\section{(B) Falling wedge pattern}

The wedge is a pattern very similar to the triangle's, being the magnitude values limited by two converging trend lines, but differs from it because both sides have a different downward inclination and often the magnitude values remain locked up in the pattern up to the apex (Figure 14).

The upper trend line crossing usually occurs between $50 \%$ and $75 \%$ of the base length.

Usually, the energy release phase consists of the mainshock preceded by an impending term foreschock (progressive earthquakes), or by the mainshock preceded by no foreshock (flash earthquakes).

Just like in the triangle pattern, the number of shock decreases during its formation.

The TM of this pattern is calculated by projecting the width of the wedge base from the breaking point (point 3).

\section{(C) Rectangle pattern}

The rectangular pattern (Figure 15 and Figure 16) is one of the easiest patterns to identify because it is characterized by fluctuations in magnitude values within a more or less extensive strip. Most likely, it represents a stage of energy accumulation before the medium-strong event.
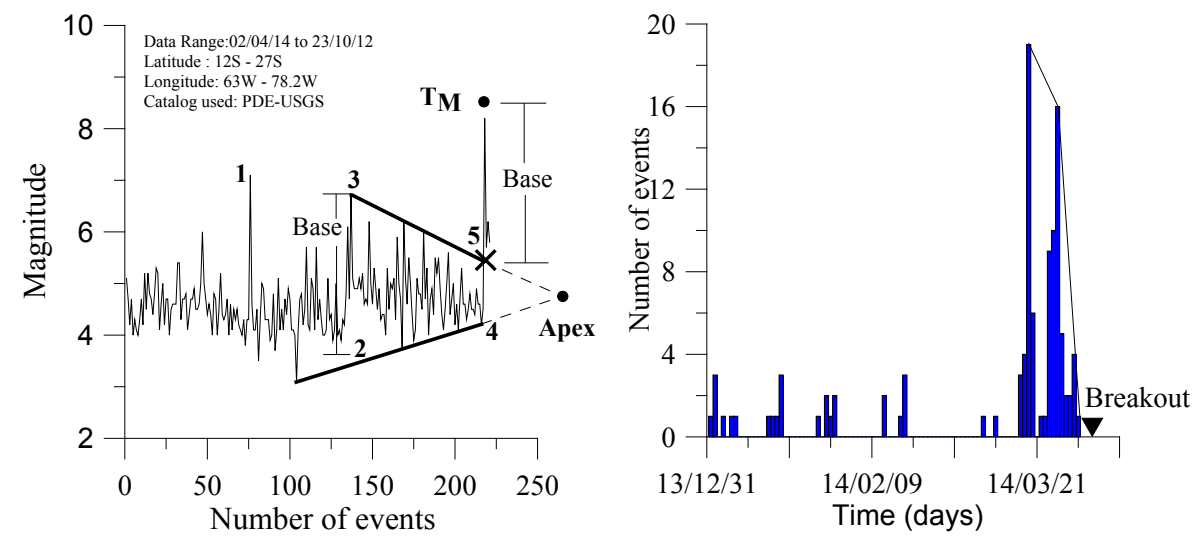

Figure 13. Symmetrical triangle pattern (Chile earthquake, 2014). 

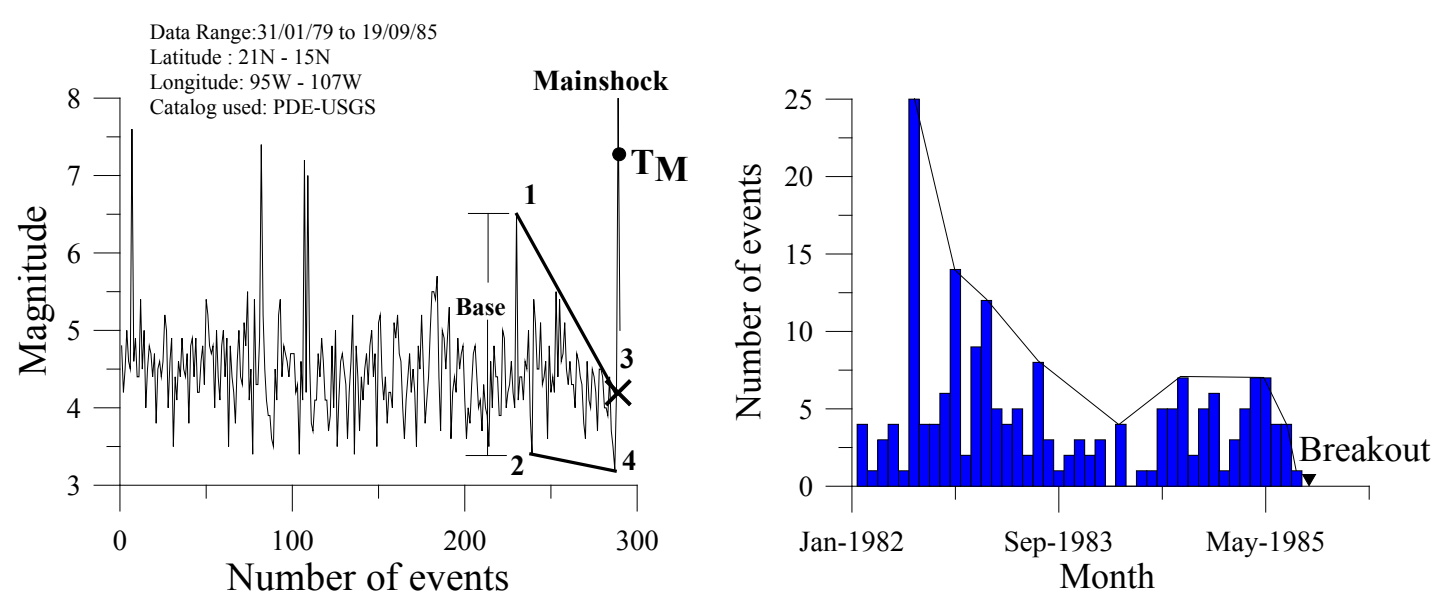

Figure 14. Falling wedge pattern (Mexico earthquake, 1985).
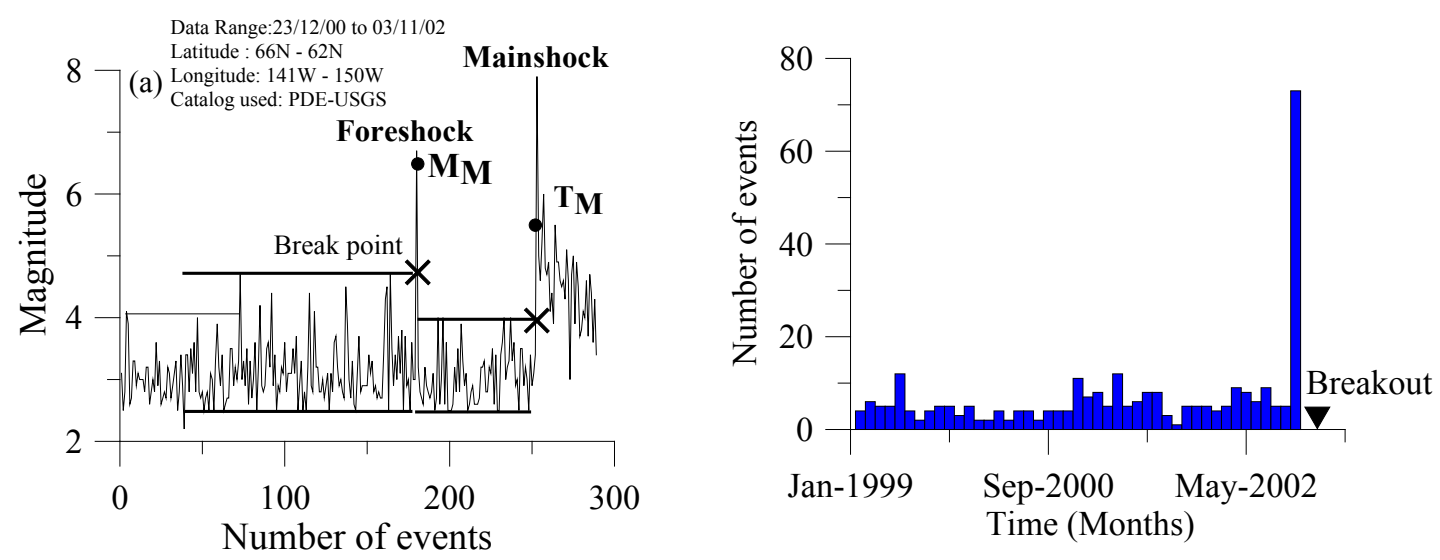

Figure15. Horizontal rectangle pattern (Denali earthquake, 2002).

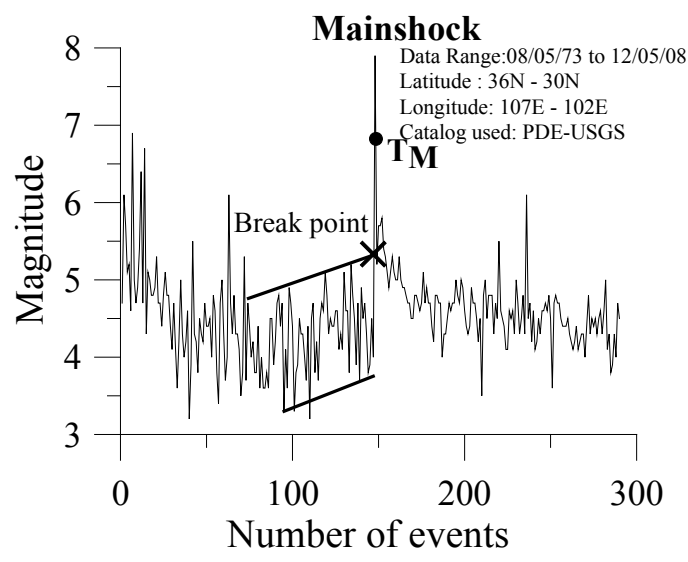

(a)

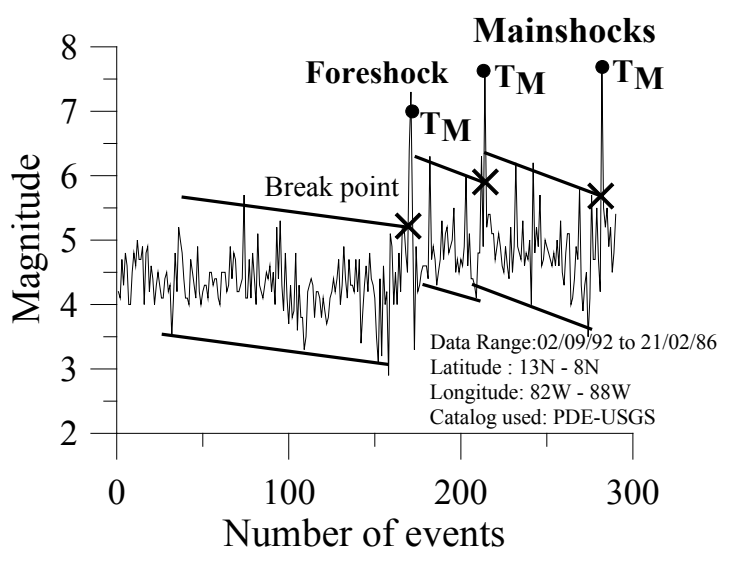

(b)

Figure 16. Rectangle Pattern: (a) ascending (China earthquake, 2008); (b) descending (Costa Rica-Nicaragua earthquakes, 1990-1992).

This pattern does not allow us to early identify the exact time when the upper trend line will be crossed.

The most frequent energy release phase consists of the mainshock preceded by no foreshock (flash earthquakes). 
Sometimes it is characterized by an increase in the number of shocks before the upper trend line crossing, as it happened, for example, during the earthquake in Denali (Alaska) coinciding with the foreshock on 23 October 2002 that preceded the earthquake on November 3rd, 2002 whose magnitude amounted to 7.9 Mw (Figure 15).

It includes three types: a) horizontal, b) ascending, c) descending, according to the arrangement of the minimum and the maximum values that are formed.

The TM of this pattern is calculated by projecting the rectangle height from the breaking point.

(D) Expansion triangle pattern

This is an uncommon pattern consisting of a series of oscillations of the magnitude minimum and maximum values that expand over time (Figure 17). The completion of the model does not coincide with the upper trend line crossing.

This pattern can be interpreted as alternate small cycles of energy accumulation and gradual release that continue until they reaches the critical stress level and, therefore, the mainshock.

The number of shock decreases during the first part of the pattern development and increases when it gets close to the breaking phase.

Pattern's special features considered, it is possible to calculate the TM at the intersection point of the line joining the maximum points with the vertical line passing from the last recorded event plus one $(n+1)$.

\section{Correlation between the Seismic Pattern and the Focal Mechanism}

The seismic patterns observed in many sequences can be correlated with the major earthquakes' focal mechanisms [14]. Table 2 shows some patterns that we could identify within the short-medium term time windows. As we see, the flash earthquakes patterns are generally associated with reverse or strike-slip faults, while progressive earthquakes patterns are related to normal faults.

Reverse faults tend to feature a higher percentage of big earthquakes not preceded by foreshocks compared to normal faults, while we have an average percentage with the strike-slip ones.

Previous studies on sequences with foreshocks showed that it would be probably very difficult to observe foreshocks before a big earthquake associated with reverse faults because normal stress would increase significantly from reverse faulting to normal [15].

Some patterns with foreshocks recorded in an environment with reverse faults seem to be associated with a greater degree of small-scale heterogeneity in crust [16].

\section{Conclusions}

With this study, we wanted to define the earthquakes triggering patterns that develop in seismic sequences before an energy earthquake that contains a series of information including the seismic history of the area under investigation.

Following a thorough graphical analysis performed over several seismic sequences, we classified the earthquakes triggering patterns according to the fluctuations of the minimum and maximum magnitude values, which in some cases may be considered as true earthquakes precursors.
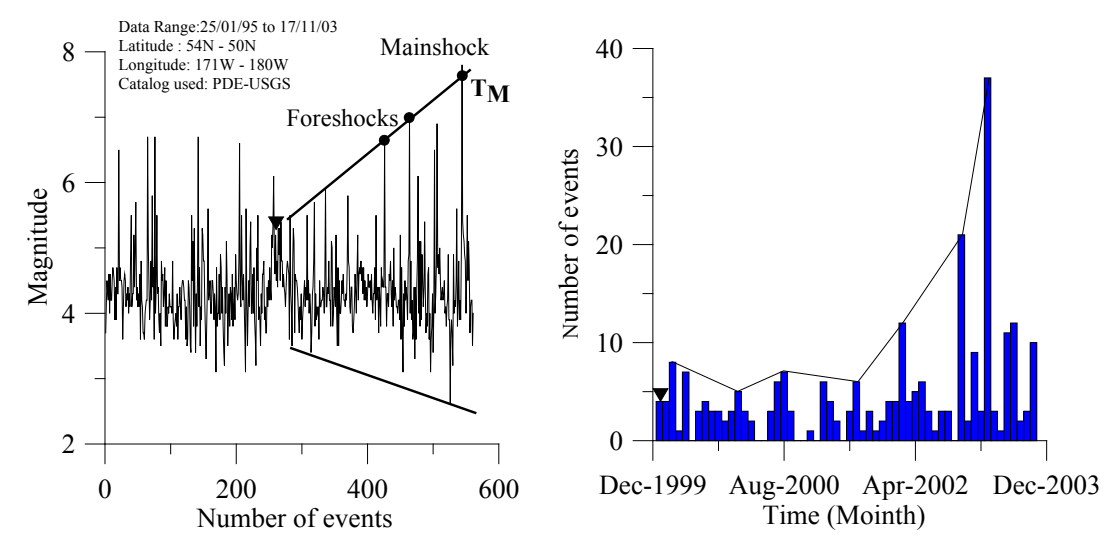

Figure 17. Expansion triangle pattern (Aleutian Islands Earthquake of 17 November 2003). 
Table 2. Earthquake pattern type and focal mechanism correlations.

\begin{tabular}{|c|c|c|c|}
\hline Earthquake & Date & Earthquake triggering patterns & Focal mechanism \\
\hline L'aquila-Italy & $06 / 04 / 2009$ & Progressive earthquakes pattern & Normal \\
\hline Colfiorito-Italy & $26 / 09 / 1997$ & Progressive earthquakes pattern & Normal \\
\hline Irpinia-Italy & $23 / 11 / 1980$ & Progressive earthquakes pattern & Normal \\
\hline Emilia-Italy & $20 / 05 / 2012$ & Progressive earthquakes pattern & Thrust \\
\hline Loma Prieta-CA, USA & 19/10/1989 & Progressive earthquakes pattern & Transpression \\
\hline Nicaragua & 02/09/1992 & Progressive earthquakes pattern & Thrust \\
\hline Vanuatu & 07/10/2009 & Progressive earthquakes pattern & Thrust \\
\hline Aleutian Islands & $17 / 11 / 2003$ & Progressive earthquakes pattern & Thrust \\
\hline Haiti & $12 / 01 / 2010$ & Progressive earthquakes pattern & Srike-slip \\
\hline Turkey & $23 / 10 / 2011$ & Progressive earthquakes pattern & Reverse strike-slip \\
\hline Taiwan & $14 / 11 / 1986$ & Progressive earthquakes pattern & Reverse \\
\hline Chile & $01 / 04 / 2014$ & Progressive earthquakes pattern & Low-angle reverse strike-slip \\
\hline Japan & $11 / 03 / 2011$ & Progressive earthquakes pattern & Thrust \\
\hline New Zealand & 03/09/2010 & Flash earthquakes pattern & Dextral strike-slip \\
\hline Landers-CA, USA & 28/06/1992 & Flash earthquakes pattern & Srike-slip \\
\hline China & $14 / 04 / 2010$ & Flash earthquakes pattern & Srike-slip \\
\hline Mexico & $19 / 09 / 1985$ & Flash earthquakes pattern & Reverse \\
\hline Pakistan & $18 / 11 / 2011$ & Flash earthquakes pattern & Normal strike-slip \\
\hline Pakistan & $24 / 09 / 2013$ & Flash earthquakes pattern & Normal strike-slip \\
\hline Nicaragua & $02 / 09 / 1992$ & Flash earthquakes pattern & Reverse \\
\hline Nicaragua & $04 / 02 / 1976$ & Flash earthquakes pattern & Srike-slip \\
\hline Uzbekistan & 19/03/1984 & Flash earthquakes pattern & Reverse \\
\hline Uzbekistan & $17 / 05 / 1976$ & Flash earthquakes pattern & Reverse \\
\hline Japan & $26 / 05 / 1983$ & Flash earthquakes pattern & Reverse \\
\hline Alaska Gulf & $30 / 11 / 1987$ & Flash earthquakes pattern & Srike-slip \\
\hline Philippines & $16 / 08 / 1976$ & Flash earthquakes pattern & Reverse \\
\hline Australia & $22 / 01 / 1988$ & Flash earthquakes pattern & Reverse \\
\hline Colombia & $15 / 11 / 2004$ & Flash earthquakes pattern & Reverse \\
\hline Colombia & 18/10/1992 & Flash earthquakes pattern & Reverse strike-slip \\
\hline Colombia & $13 / 07 / 1974$ & Flash earthquakes pattern & Low-angle reverse \\
\hline Indonesia & $11 / 04 / 2012$ & Flash earthquakes pattern & Low-angle reverse \\
\hline Indonesia & $06 / 04 / 2010$ & Flash earthquakes pattern & Srike-slip \\
\hline Sumatra & $26 / 12 / 2004$ & Flash earthquakes pattern & Thrust \\
\hline Nepal & $25 / 04 / 2015$ & Flash earthquakes pattern & Reverse \\
\hline Emilia-Italy & $29 / 05 / 2012$ & Flash earthquakes pattern & Reverse \\
\hline Friuli-Italy & $06 / 05 / 1976$ & Flash earthquakes pattern & Reverse strike-slip \\
\hline Denali (Alaska) & $03 / 11 / 2002$ & Flash earthquakes pattern & Reverse strike-slip \\
\hline Tonga & $03 / 05 / 2006$ & Flash earthquakes pattern & Srike-slip (transform) \\
\hline Russia & $05 / 12 / 1997$ & Flash earthquakes pattern & Srike-slip (transform) \\
\hline Australia & $22 / 01 / 1988$ & Flash earthquakes pattern & Reverse \\
\hline Uganda & $05 / 12 / 2005$ & Flash earthquakes pattern & Transtensive \\
\hline Taiwan & 20/09/1999 & Flash earthquakes pattern & Reverse \\
\hline
\end{tabular}


The statistical studies performed on seismic sequences worldwide demonstrated that the earthquakes triggering patterns, in relation to the evolution usually shown over time, tend to group into two types: 1) flash earthquakes patterns; 2) progressive earthquakes patterns.

By considering the focal mainshock mechanisms, we saw how the earthquakes triggering patterns, associated with reverse and strike-slip faults tend to have a higher percentage of big earthquakes of flash earthquakes patterns type compared to normal faults that develop earthquakes of progressive earthquakes patterns type.

Some patterns featuring foreshocks recorded in an environment with reverse faults seem to be associated with a greater degree of small scale crustal heterogeneity and maybe even to a reduced normal stress on the fault plane.

The aforementioned approach unquestionably has some advantages in terms of prediction. In fact, the early detection of the earthquakes triggering patterns, allows us not only to predict the likely future changes in magnitude values but also to graphically estimate the magnitude of the strongest event.

\section{References}

[1] Jiao, M., Tang, C., Zhang, G., Shi, Y. and Hou, W. (2003) A Numerical Test on Influence of Mesoscopic Heterogeneity on Macroscopic Behavior of Rock Failure and Seismic Sequance Types. Chinese Journal of Geophysics, 46, 943-953. http://dx.doi.org/10.1002/cjg2.414

[2] Matsu'ura, R.S. (1986) Precursory Quiescence and Recovery of Aftershock Activities before Some Large Aftershocks. Bulletin of the Earthquake Research Institute, 61, 1-65.

[3] Matsu'ura, R.S. (1991) Case 1: Precursory Quiescence and Recovery of Aftershock Activity before Some Large Aftershocks. In: Wyss, M., Ed., Evaluation of Proposed Earthquake Precursors, American Geophysical Union, Washington DC, 8-11. http://dx.doi.org/10.1029/SP032p0008

[4] Phetking, C., Sap, M.N.M. and Selamat, A. (2008) Time Series Representation for Elliott Wave Identification in Stock Market Analysis.

http://comp.utm.my/pars/files/2013/04/Time-Series-Representation-for-Elliott-Wave-Identification-in-Stock-Market-A nalysis.pdf

[5] Frost, A.J. and Prechter, R.R. (1985) Elliott Wave Principle. New Classics Library, Georgia.

[6] Prechter Jr., R.R. (1995-2004) The Basics of the Elliott Wave Principle. New Classics Library, Gainesville. http://www.yinyangforex.com/download/trading_books_and_documents/EW\%20Basics\%20Booklet.pdf

[7] Bulkowski, T.N. (2002) Trading Classic Chart Patterns. John Wiley \& Sons, Inc., Hoboken. http://fx-arabia.com/vb/uploaded/178 01275676445.pdf

[8] Matras, K. (2010) Chart Patterns Trader Supplement Understanding and Trading Classic Chart Patterns. Zacks Investment Research, Inc. http://www.sju.edu/int/academics/hsb/finance/wstr/pdf/CPGuide1.pdf

[9] Mogi, K. (1963) Some Discusions on Aftershocks, Foreshocks and Earthquake Swarms: The Fracture of a SemiInfinite Body Caused by Inner Stress Origin and Its Relation to the Earthquake Phenomena (3rd Paper). Bulletin of the Earthquake Research Institute, 41, 615-658.

[10] Chen, Y.T. and Knopoff, L. (1987) Simulation of Earthquake Sequences. Geophysical Journal International, 91, 693709. http://dx.doi.org/10.1111/j.1365-246X.1987.tb01665.x

[11] Riguzzi, F., Crespi, M., Devoti, R., Doglioni, C., Pietrantonio, G. and Pisani, A.R. (2012) Geodetic Strain Rate and Eartquake Size: New Clues for Seismic Hazard Studies. Physics of the Earth and Planetary Interiors, 206-207, 67-75. http://dx.doi.org/10.1016/j.pepi.2012.07.005

[12] Scholz, C.H. (2002) The Mechanics of Earthquakes and Faulting. 2nd Edition, Cambridge University Press, Cambridge, UK. http://dx.doi.org/10.1017/cbo9780511818516

[13] Abercrombie, R.E. and Mori, J. (1996) Occurrence Patterns of Foreshocks to Large Earthquakes in the Western United States. Nature, 381, 303-307. http://dx.doi.org/10.1038/381303a0

[14] Global CMT Catalog Search. http://www.globalcmt.org/CMTsearch.html

[15] Sibson, R.H. (1974) Frictional Constraints on Thrust, Wrench and Normal Faults. Nature, 249, 542-544. http://dx.doi.org/10.1038/249542a0

[16] Lin, C.-H. (2004) Repeated Foreshock Sequences in the Thrust Faulting Environment of Eastern Taiwan. Geophysical Research Letters, 31, L13601. http://dx.doi.org/10.1029/2004GL019833 